\title{
Gasos Guínicos \\ Manejo despigmentante de vitiligo generalizado con monobenzil eter de hidroquinona
}

\author{
Raúl Charlín ${ }^{1}$, Karen Valenzuela ${ }^{2}$
}

\section{RESUMEN}

El vitiligo es una patología crónica, recidivante y difícil de tratar. El objetivo primario del tratamiento es inducir la repigmentación, sin embargo, en casos extensos refractarios a tratamiento, se puede realizar despigmentación para corregir la discromía. Dentro de los tratamientos despigmentantes en vitiligo, a la fecha el único aprobado por la FDA es el Monobenzil éter de hidroquinona (monobenzona). Se expone el caso de una paciente con vitiligo extenso y refractario a tratamiento que fue manejado con monobenzona. El resultado fue exitoso durante tiempo prolongado, con recaída parcial al suspender el medicamento. La recaída remitió con el reinicio de la monobenzona. Sin nueva recaída actualmente con tratamiento de mantención 3v/semana. La monobenzona induce acromía secundaria a necrosis de los melanocitos. Se requiere su uso 1 a 2 veces al día por 6-12 meses para lograr la despigmentación. En pacientes adecuadamente seleccionados, es una alternativa válida para el manejo del vitiligo. Se presenta un caso exitoso de despigmentación con monobenzona. Actualmente, la paciente está muy satisfecha con los resultados.

Palabras claves: Vitiligo; despigmentación; monobenzona.

\section{SUMMARY}

Vitiligo is a chronic, recurrent pathology, difficult to treat. The primary goal of treatment is to induce repigmentation, however, in extensive cases refractory to treatment depigmentation of surrounding skin may be performed to correct the cosmetic misbalance. To date the only depigmenting treatment for vitiligo approved by the FDA is the hydroquinone monobenzyl ether (monobenzone). We report the case of a patient with extensive vitiligo refractory to treatment managed with monobenzone. The result was successful for a long time, with partial relapse when the drug was discontinued. The relapse ended with the restart of the monobenzone. No new relapse seen with maintenance treatment 3 times a week. Monobenzone induces acromy due to melanocyte necrosis. To achieve depigmentation, it is used 1 to 2 times a day for 6 to 12 months. In adequately selected patients, it is a valid alternative for the management of vitiligo. A successful case of monobenzone depigmentation in dyschromia due to extensive vitiligo. Patient currently very satisfied with the results.

Key words: Vitiligo; despigmentation; monobenzone.
$\mathrm{E}$ l vitiligo es un patología adquirida que se produce por destrucción de los melanocitos de la epidermis interfolicular y ocasionalmente del folículo piloso ${ }^{1}$. Clínicamente se presenta como máculas acrómicas que pueden estar tanto en la piel como el pelo. Afecta aproximadamente al 0,5 a $1 \%$ de la población, con mayor frecuencia antes de los 20 años ${ }^{2}$. La despigmentación puede ser causa de gran estrés psicológico, sobre todo en pacientes de fototipos altos. Los tratamientos actuales intentan detener la progresión de la enfermedad e indu-

'Dermatólogo. Servicio Dermatología, Hosptital Barros Luco. Santiago, Chile. ${ }^{2}$ ResidenteDermatología / Magister en nutrición. Departamento de Dermatología, Hospital Clínico Universidad de Chile. Santiago, Chile.

Correspondencia: Karen Valenzuela L Correo electrónico: drakarenvalenzuela@gmail.com Celular: +56 9 75174075. Dirección: Santos Dumont 999. Independencia, Santiago, Chile. Código Postal: 8380456 . cir repigmentación o, en casos excepcionales, disminuir la pigmentación para lograr resultados cosméticamente agradables y mejorar la autoestima y calidad de vida del paciente $^{3}$. El tratamiento despigmentante debe considerarse en vitiligo extenso, refractario y desfigurante, vitiligo facial recalcitrante o de manos muy visible ${ }^{4}$. Sin embargo, es una modalidad de tratamiento más agresivo, de carácter generalmente irreversible, por lo cual debe ser evaluado en base a cada individuo.

El objetivo del presente caso es reportar el tratamien- 
to exitoso de despigmentación en paciente con vitiligo crónico, con el uso de monobenzil éter de hidroquinona (monobenzona) al 20\%.

\section{GASO GLÍNICO}

Paciente de 44 años, con antecedentes de hipotiroidismo en tratamiento con levotiroxina $175 \mathrm{mg} /$ día. Historia de aproximadamente 15 años de vitiligo con despigmentación progresiva.

$\mathrm{Al}$ examen físico presenta acromía de alrededor del $80 \%$ de la superficie corporal, con excepción parcial del rostro, que además presenta zonas de hipercromía (Figura $1)$.

Se decide, en conjunto con equipo médico y paciente, la despigmentación del rostro con monobenzona $20 \%$, elaborada en recetario magistral. Se indica aplicación facial en noches alternadas por 2 semanas y luego todas las noches. Además se inicia fotoprotección estricta.

La paciente evoluciona con despigmentación progresiva del rostro. Al año de aplicación se observa despigmentación completa, pero repigmenta parcialmente tras la suspensión del tratamiento. Al reiniciarlo vuelve a la acromía, que se ha mantenido por 5 años con aplicación de mantención en días alternados de receta magistral de Monobenzil éter de hidroquinona 15\% y fotoprotección rigurosa.

$\mathrm{Al}$ examen físico en el último control se observa piel de rostro y cuello con despigmentación uniforme (Figura 2 y 3). Se reduce dosis de Monobenzil éter de hidroquinona al 10\% con aplicación solo 2 veces a la semana.

Paciente muy conforme con los resultados.

\section{Discusión}

Existen múltiples métodos de despigmentación permanente establecidos tales como la crioterapia, terapia láser, solución de fenol 88\%, monometil éter de hidroquinona y monobencil éter de hidroquinona ${ }^{5}$.

El monobencil éter de hidroquinona es un fenol que genera una muerte necrótica de melanocitos epidérmicos. Además se ha descrito que induce inmunidad celular contra melanocitos, lo que explicaría episodios ocasionales de despigmentación a distancia y un eventual uso como inmunoterapia contra melanoma ${ }^{6}$.

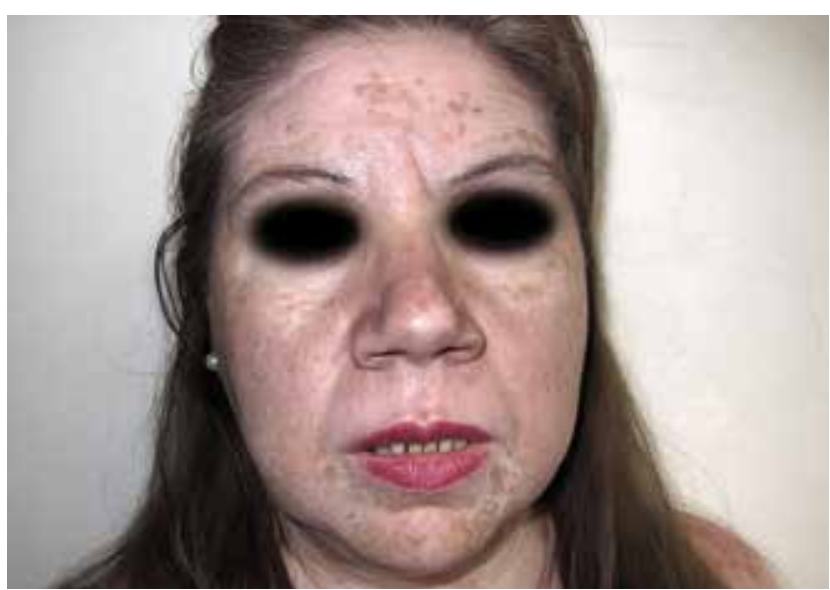

Figura 1

Vitiligo. Paciente pre tratamiento

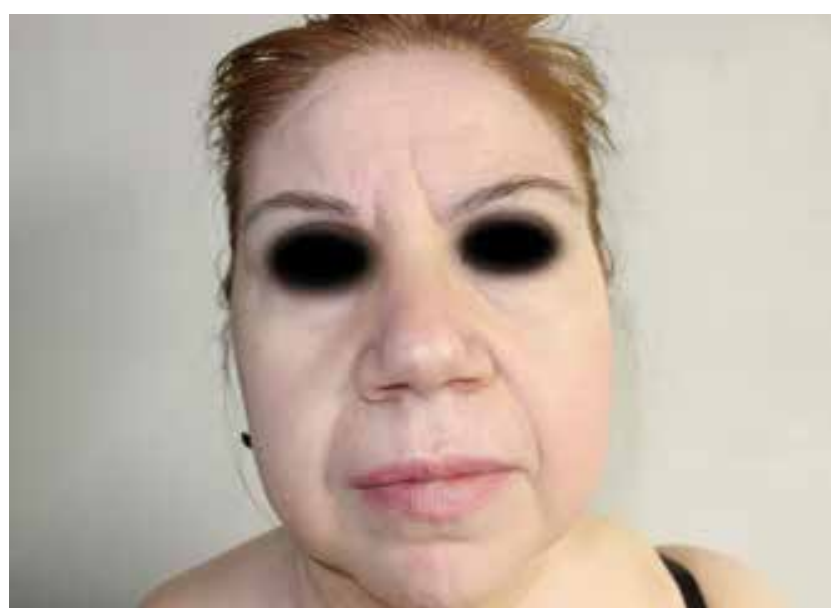

Figura 2 y 3

Vitilgo. Paciente post tratamiento

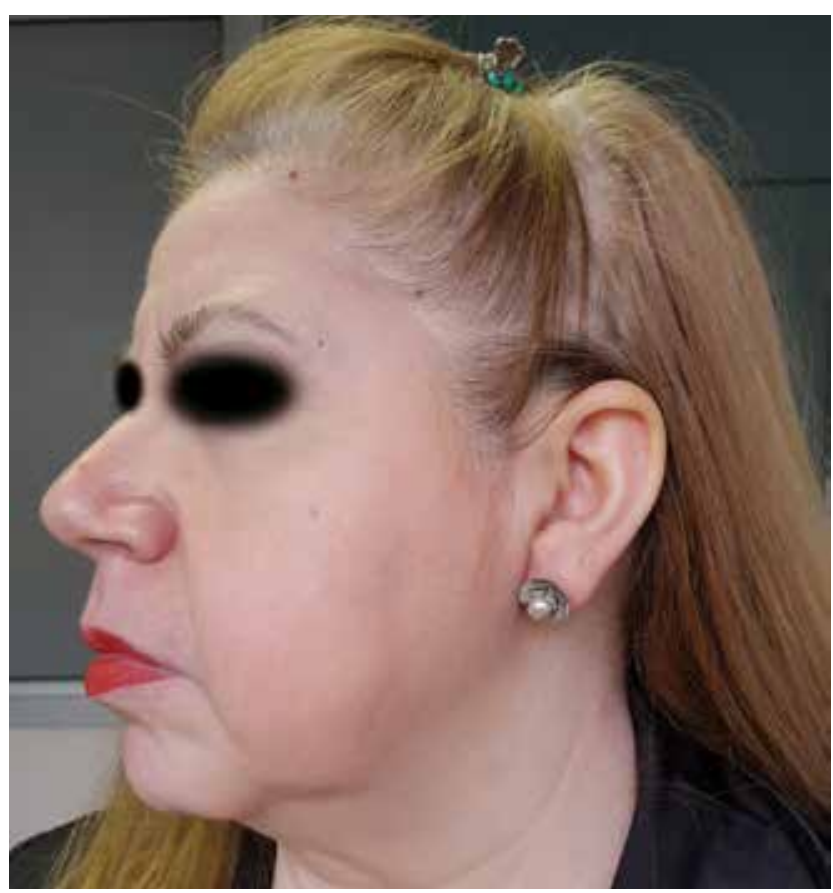


El primer reporte de monobenzona como despigmentante fue en 1939 por Oliver et al., quienes describieron despigmentación de manos en trabajadores que usaban guantes de goma con monobenzona como antioxidante ${ }^{7}$.

La monobenzona fue aprobada por la FDA en el año 1952 como agente despigmentante en el tratamiento del vitiligo extenso, y a la fecha sigue siendo el único ${ }^{5}$.

En el año 1977 Mosher y cols., reportan unos de los primeras de casos de tratamiento exitoso con monobenzona al 20\% en 11 de 18 pacientes con vitiligo, luego de 10 meses de tratamiento ${ }^{8}$.

Aunque no hay consenso en la literatura, creemos que el cuadro debe estar inactivo por al menos 1 año para escoger esta opción terapéutica.

La monobenzona se utiliza en concentración al 20\%, cada 12-24 horas, pero se sugiere su inicio en una concentración menor y/o pruebas de tolerancia extrafaciales previo al tratamiento. El efecto de la droga es irreversible y comienza a verse al mes de iniciada la terapia, sin embargo se requieren entre 6 a 12 meses de uso continuo para lograr acromia ${ }^{9}$. Si la despigmentación no ocurre a los 3 a 4 meses de iniciada la terapia a una concentración al 20\%, ésta puede incrementarse hasta un $40 \%$ como máximo, pues concentraciones más altas no son recomendadas ${ }^{5}$. Su efecto acostumbra ser permanente, pero tal como en el caso de nuestra paciente, se ha reportado repigmentación a las pocas semanas luego de descontinuar una terapia exitosa con monobenzona, con repigmentación más oscura que la piel original y en áreas previamente afectadas con vitiligo ${ }^{10}$, lo cual podría explicarse por hiperactivación de los melanocitos y una sobrevida de ellos a pesar del tratamiento ${ }^{10}$. Se ha observado además repigmentación secundaria a la exposición solar, por lo cual es mandatoria una adecuada fotoprotección. En este caso en particular, la repigmentación ha sido manejada exitosamente con terapia de mantención con monobenzona a menor concentración en forma trisemanal y una rigurosa protección a la exposición de la radiación solar.

Se describe que la asociación con un ácido retinoico potenciaría su efecto despigmentante, comenzando a verse despigmentación entre las 4 y 8 semanas. Se indica que el retinoide aumenta la penetración en la piel del despigmentante y disminuye la melanogénesis ${ }^{5}$.

Entre los principales efectos adversos de la monobenzona se encuentran la dermatitis de contacto irritativa, efectos adversos oculares, ocronosis exógena, la dificul- tad en predecir su respuesta ${ }^{5}$ y un eventual rebote pigmentario al interrumpir tratamiento.

\section{Conclusión}

Se presenta un caso exitoso de manejo despigmentante con monobenzona al $20 \%$ en paciente con discromía secundaria a vitíligo extenso, con recaída parcial luego de su suspensión, pero con respuesta favorable con su reinicio como tratamiento de mantención.

La despigmentación permanente en el manejo del vitiligo no es una terapia de primera línea y debe ser indicada previa información precisa y debida aprobación del paciente. En casos específicos, es una herramienta de gran valor para mejorar la calidad de vida del paciente.

\section{REFERENCIAS}

1. Chan MF, Chua TL. The effectiveness of therapeutic interventions on quality of life for vitiligo patients: A systematic review. Int J Nurs Pract. 2012;18(4):396-405

2. Alikhan A, Felsten LM, Daly M, Petronic-Rosic V. Vitiligo: A comprehensive overview: Part I. Introduction, epidemiology, quality of life, diagnosis, differential diagnosis, associations, histopathology, etiology, and work-up. J Am Acad Dermatol. 2011;65(3):473-491

3. Felsten LM, Alikhan A, Petronic-Rosic V. Vitiligo: A comprehensive overview: Part II: Treatment options and approach to treatment. J Am Acad Dermatol. 2011;65(3):493-514

4. Ezzedine K, Eleftheriadou V, Whitton M, Van Geel N. Vitiligo. Lancet. 2015;386(9988):74-84

5. AlGhamdi KM, Kumar A. Depigmentation therapies for normal skin in vitiligo universalis. J Eur Acad Dermatology Venereol. 2011;25(7):749-757

6. Van Den Boorn JG, Melief CJ, Luiten RM. Monobenzone-induced depigmentation: From enzymatic blockade to autoimmunity. Pigment Cell Melanoma Res. 2011;24(4):673-679

7. Oliver E, Schwartz L., Warren L. Occupational leukoderma: preliminary report. Oliver E, Schwartz L, Warren, L. 1939;113:927

8. Mosher DB, Parrish JA FT. Monobenzylether of hydroquinone. A retrospective study of treatment of 18 vitiligo patients and a review of the literature. BrJ Dermatol. 1977;97(6):669-679

9. Soria LM, Spelta G, Garrido A, Chames C, Chouela E. Vitiligo universal . Tratamiento despigmentante con luz pulsada intensa Vitiligo universalis . Dermatol Argent 2010;16 Supl 2:34-36

10. Oakley AMM. Rapid repigmentation after depigmentation therapy : Vitiligo treated with monobenzyl ether of hydroquinone. 1996;(1995):96-99 